\title{
Shifting Concepts of Genetic Disease
}

\author{
Sara Melendro-Oliver
}

\begin{abstract}
For many years the rhetoric of the new genetics have been criticised for their inherent determinism, especially in the area of health. The move from genetics to genomics has meant that more than just individual genes will be looked at in the causation of disease. At the same time, the findings from the Human Genome Project have challenged the deterministic assumption of the one gene - one trait tenet. The concept of genetic disease, however, is still predominant and still expanding to include more conditions every day under its name. Here, l look at how the model of genetic causation of disease or what I have called the 'gene model' is becoming dominant and how this underlines a process of geneticisation, which does not seem to have stopped under the genomic perspective.
\end{abstract}

Keywords: genetic disease, genomics, determinism.

Twenty years ago Yoxen (1984) drew attention to the construction of the idea of genetic disease and how this played an important role in the medical and cultural expansion of molecular genetics. Yoxen drew attention to the determinism and reductionism involved in seeking to explain the nature and causes of many diseases in genetic terms. She argued that genetic disease had become a very large category "encompassing not only genetic disorders that are thought of as diseases but also genetic abnormalities associated with no known disorder as well as disorders that may be neither genetic nor diseases" (Yoxen, 1984: 49). Only a few years after critiques like Yoxen's were launched, the concept of 'genomics' was introduced into the language of molecular biology and a couple of years later its biggest ever endeavour, the Human Genome Project (HGP) started. Genomics and the HGP, as will be argued, were to provide the information for more complex explanations of the relationship between genes and health. The findings of the much lower than expected number of genes in the human genome sequence supported those who had challenged the reductionism of the one gene- one trait model. Scientists and journalists, according to their statements, seemed to agree that these results challenged de- 
terminism. Thus, has the discovery, shocking to many that we could not point towards a gene for every ailment we suffer and every deviation we show changed the way we are looking at genetics? Moreover, as mentioned above, genetics was not only criticised for being deterministic but also for its reductionism, and in fact these two critiques went hand in hand. Genetics was considered reductionist in its disregard for other factors outside genes (notoriously in the area of medicine) and deterministic in conferring so much causal power to the gene. Accordingly, once it was discovered that there are not enough genes to sustain the deterministic tenet, the implication for the reductionist critique is clear; if there are not enough genes to cause every trait, then other factors have to be involved, reductionism was also called into question. So, have these challenges produced a change in how we understand and conduct genetics? Has this new understanding challenged the power of the gene?

In this paper I seek to partially address these questions by looking at how the concept of genetic disease has evolved in relation to these challenges. I will develop my argument by examining the concept of genetic disease within each of the three assumptions that Dubos highlighted in 1959 as the basic tenets of the 'germ model' and draw a parallel between this and what I have accordingly called the 'gene model'. I will look at how the current use of the term genetic disease features for each one of these assumptions, using examples from journalistic and scientific texts to illustrate my point.

\section{Genomics and the Human Genome Project}

In 1986 Thomas Roderick introduced the term 'genomics' and later went on to found the journal that carries the same name (Hieter and Boguski, 1997). The Human Genome Project (HGP) was conceived around the same time and officially started in 1990. Guttmacher and Collins explain the difference between genetics and genomics as follows:

Genetics is the study of single genes and their effects. 'Genomics,' (...) is the study not just of single genes, but of the functions and interactions of all the genes in the genome. Genomics has a broader and more ambitious reach than does genetics (Guttmacher and Collins, 2002: 1512).

Genomics' broader perspective offered the possibility of creating a more complex picture than the 'one gene- one trait model' underlying genetics and which was the basis of many of the critiques against it, especially in the area of health. Genomics' major enterprise, the HGP, involved a qualitative move towards more complex explanations. When data started to come from the HGP, ErnstLudwig Winnacker (acting president of the Deutsche Forschungsgemeinschaft), emphasised this qualitative move when he claimed:

Until know, the individual genes stood in the foreground. We will leave them behind us and ask how they contribute to the formation of individual cells, of cell communities, and of whole organisms... We will go for an understanding of the whole" (Winnacker 1997, quoted in Thieffry and Sarkar, 1999: 223). 
Ricki Lewis, contributing editor for The Scientist, is describing the process she went through when writing a book on genetics, and she explains:

As I began rewriting, I quickly realized that "genomics" is not just a new buzzword, but an entirely different way of thinking about biology. Genetics is no longer based on a one gene - one enzyme, or even a "this mutation causes that disease" paradigm. Instead we now view a genome as a dynamic entity, a vast storehouse whose information is accessed in staggeringly complex panoply of expression exquisitely controlled in time and place throughout a life (Lewis, 2000: 46).

Some, however, did not seem to share neither Winnacker's enthusiasm nor Lewis' optimism and argued that the determinism inherent in the priority of genetic explanations was not only still alive in the HGP but was in fact helping to promote it (see Keller, 1992; NeumannHeld, 2001; Lindee, 2002). Following Yoxen's analysis, Keller (1992) argued that the concept of genetic disease created the climate in which the HGP could appear both reasonable and desirable by the constant emphasis on how this project will allow the diagnosis, treatment and prevention of human disease. Moreover, she stressed that this represented an ideological expansion of molecular biology far beyond its technical successes (Keller, 1992: 293).

Nevertheless, the biggest challenge to determinism, at least in its simpler version, was going to come not from social scientists, but from the results of the Human Genome Project itself. Although the critiques of determinism launched against genetics had started long before, it was when the HGP was published that these claims were strongly backed by the very information arising from genetics' biggest ever project. The HGP showed that the whole sequence of our genome only contained 30,000 genes, nowhere near the number needed to support the deterministic tenet of one gene - one trait. Genetic determinism appeared flawed and was no longer sustainable.

Both scientists and journalists acknowledged the significance of the low number of genes that the HGP has revealed. Judging by the declarations following the announcement, the challenge was duly accepted by those in charge of the HGP. An article in The Independent on 12 February 2001, the day of the announcement of the working draft of the genome project, quotes Dr. Venter saying that this finding (the low number of genes) "is a body blow to the common fallacy that single genes determine all human traits". Another article the same day, this time in The Guardian, argues that "the vastly smaller number of genes means that biologists can no longer assume that one gene is a blueprint for one protein that has one function" and further down on the article they reiterate this idea that "[the findings] rule out another habit of thinking called genetic determinism: the argument that humans might be little more than robots controlled by their genes".

Despite the strong claims of the 'death of determinism' appearing everywhere after the first draft of the HGP, reductionism and determinism in the world of molecular biology, and especially in the area of health does not seem to have been overcome. Genomics, if certainly looking at a more complex picture than individual genes, does still retain a reductionist and deterministic perspective where genes are still the 'key' 
to our health and illness and other factors are considered secondary. One of the best ways to observe this is by looking at the evolution of the concept of genetic disease and how it is still used to prioritise genetic explanations.

\section{Disease as a Construction}

As both the history of medicine and medical sociology show, many diseases that were diagnosed frequently in the past are no longer recognized as diseases and new disease concepts arise that previously did not exist (Freund and McGuire, 1995). Moreover, not only what is considered a disease varies across time and space, but also the explanation of their aetiology keeps changing. In relation to this, Freund and McGuire (1995: 193) argue that 'the forces shaping the "discovery" of disease categories are not purely objective, scientific factors; rather, value judgements, economic considerations, and other social concerns frequently enter the process.'

During most of the $20^{\text {th }}$ century, the 'germ theory' model dominated aetiological explanations of disease (Conrad, 1999). The prevalent model now, I shall argue, is a 'gene model' where new genetic diseases are constantly appearing and many common diseases that already existed are being re-defined in genetic terms. The concept of genetic disease has not remained stable but has been changing along with the development of molecular biology. The constant search for genes, together with the proliferation of genetic tests and gene therapy (or its promises) has expanded the category of genetic disease to include most common acquired diseases. As mentioned earlier, however, this con- ceptual change in the aetiology of many diseases and the expansion of the category of genetic disease is only in part explicable by alluding to the development of scientific knowledge. In Health, Illness, and the Social Body (1995), Freund and McGuire argue that funding for research is largely dependent upon what is socially defined as a serious problem at the moment. Accordingly, the more emphasis that is given to the genetic component of diseases, the more likely the professional area of science studying this (namely, molecular genetics) would be to get funding. 'Often the development of new disease categories is connected with the assertion of a new occupational specialization' (Freund and McGuire, 1995: 196). Similarly, Petersen (2002: 133) argues that scientists have a vested interest in highlighting the benefits of their work, and may emphasize particular diseases and their "causes" as part of a persuasive strategy to convince people to focus on certain therapies or approaches. Accordingly, as Neumann-Held (2001:11) argues, from the very beginning the hopes attached to the human genome project, centred around the promise of getting a better grip on human diseases.

If the extent to which biotechnology and genetics have done this is still debated, what they have clearly done is to familiarise us with the idea of genetic disease. This concept was previously unheard of or only related to strange and rare conditions, but it is today a familiar notion that we keep encountering in media reports on everything from cancer to obesity. While it is difficult to deny the fact that the media uses hype and sensational statements to get the message across, the idea that just the media is to blame for 
the determinism and reductionism of these reports is mistaken. Scientists and researchers on genetics have used the media to promote their work and boost public expectation about possible cures in order to gain support and funding for their projects (Nelkin, 1985; Van Dijk, 1998).

The growing geneticization of health and illness, assumes that most disorders have a genetic cause and makes the concept of genetic disease every day more common in both public and scientific discourse. David Magnus (n/d) argues that the definition of a genetic disease has changed from the fairly restricted notion of a disease traceable to a single locus defect with $100 \%$ heritability, to a present extended usage where it represents common disease and even complex behavioural traits such as alcoholism. Certainly, during the last 30 years the category of genetic disease has grown continually. Today, conditions labelled as genetic are so many and various that subcategorise have arisen. Those now called single-gene disorders or mono-genetic diseases are around 4,000; others described as polygenic diseases have not even been counted. These two subcategories are of course based in the obvious difference of one gene or multiple genes forming part of (or being, depending where you read) the causes of the disease. A third category is that of 'multifactorial', indicating the fact that other factors besides genes are involved in the causation of the disease but still placing it under the rubric of genetic. The contradiction in terms of referring to a disease as genetic and multifactorial does not seem to have struck anyone. Moreover, the creation of new subcategories (besides the term ge- netic disease) to include new disorders when there was no need to reclassify them, such as cancer, heart disease or diabetes is significant.

There is no doubt that in many of these diseases a genetic component is involved, however, they could have been studied just the same without having to create new labels. Labelling them polygenic or multifactorial allowed them to be classified within the genetic category while avoiding the fact that they do not fit the Mendelian model, which was the basis of the genetic disease category. When these common acquired diseases started to be talked of as genetic, obvious inconsistency arose since the concept was until then only used for monogenic conditions which were simply called 'genetic'. Therefore this concept was split; genetic diseases became 'single gene disorders' or monogenic and constituted one aspect of molecular pathology as opposed to being the subject of the new genetics. Everything else for which any genetic factor can be found has been added to either the polygenic or the multifactorial categories. Illnesses such as diabetes, asthma, hypertension and cancer are now routinely referred to as genetic diseases by many and not only in the media, but also by scientists themselves.

Human genetic diseases are ubiquitous. Some are prevalent-such as diabetes, asthma, hypertension and cancer-but many are rare, with incidence at or less than one person per 10,000 live births" (Scriver, 2001: 113).

As we can see here, another way of differentiating between genetic diseases is the seemingly less scientific division between 'common' and 'rare' genetic diseases that in great part coincide with the 
polygenic/multifactorial and monogenic categories respectively. Genetic disease is turning from being a disease category (in itself a problematic concept) into becoming an explanation model for all types of diseases (crossing across different categories), a model of the causal explanation of disease itself. Any type of disease seems to be open to the genetic gaze, no matter what category it is.

A new gene model for explaining disease aetiology is emerging following a similar path to other previous theories, such as the germ theory (notion that microbes cause disease) and becoming, as the germ theory previously did, the dominant explanation of disease causation. Conrad explains how the successful 'germ theory' model for explaining disease was based fundamentally on three assumptions that were highlighted by Dubos (1959): the doctrine of specific aetiology; the focus on internal rather than external environment; and the metaphor of the body as machine. The structure of popular conceptions of the new genetics shows remarkable parallels with germ theory. I will now look at these three assumptions in more detail and relate them to the concept of genetic disease.

\section{Doctrine of Specific Aetiology}

The assumption of specific aetiology can still be seen operating in the gene model, where the narrative is moulded so that the specific agent causing disease is no longer a micro organism, but a gene or set of genes. This narrative has pervaded public discourse about the origin and development of disease. Increasingly genes are seen not only as causing dis- ease, but as the very locus of disease. Moreover, the gene model goes even further than previous ones, in that not only does it place genes as causal explanants of disease, but it also creates a discourse involving a shift in the criteria of what constitutes illness, from symptoms of unhealth as experienced by an individual patient to whatever is revealed in his or her DNA sequences.

The popular conception of genetic disease whereby disease is seen to be a product of a genetic defect governs news media portrayals and reinforces the view that quick fixes and personal risk management strategies rather than broad social structural and environmental solutions are needed to prevent illness or restore health (Petersen, 2002). The disease comes to be seen as a feature or property of the patient, derived from her/his genetic material, as something the patient 'has' (on the gene model, the person is represented as having a faulty gene). This reified disease identification often assumes primacy; if it conflicts with the patient's subjective illness experience, the objectified disease-thing is often treated as more real than the sick person's feelings. This is very patent especially in genetic diagnoses for future illnesses (the so-called pre-symptomatic diagnoses), where the patient has no symptoms but the diagnoses takes primacy over the healthy feeling of the individual.

Although some monogenic diseases might be said to function like this, they only account for about $2 \%$ of the total disease load (Strohman, 2000). The specific aetiology tenet, however, seems to slide from single-gene disorders to any other condition that is in one way or another, referred to as genetic. In 1999, in 
a lecture on the medical and societal consequences of the HGP, the director of the Human Genome Research Institute, Francis Collins, explained that amongst other things, the benefits from mapping the human genome "would include a new understanding of genetic contributions to human disease and the development of rational strategies for minimizing or preventing disease phenotypes altogether" (1999: 29). But, it is only if we adopt a very narrow view of disease aetiology and a deterministic relationship between genotype and phenotype that we can conceive of 'preventing disease phenotypes all together' by the mapping of the human genome.

The specific aetiology assumption in genetics was deeply shaken, as we mentioned earlier, by the completion of the HGP and its 'shocking' finding that the human genome possesses just 30,000 genes instead of the more than 100,000 expected. This obviously meant that the one-gene-one-protein-one function model was definitely no longer sustainable. The culminating moment of the genomic era, the completion of the HGP, brought to light with its release the failure of genetic determinism. The reductionist assumption that complex characteristics can be pinned down to bits of genetic information was challenged by the results of the HGP, nevertheless this does not seem to have stopped those who are eager to find them. Strohman explains this occurrence powerfully, so I quote him here at length;

But after almost a century of life sciences dominated by this theory [genetic determinism], and after ten years of the Human Genome Project (HGP) dedicated to finding the genes which cause human disease, with the human genome finally sequenced and biotechnologists and drug companies standing by - after all, to announce that the entire project was based on an incomplete and flawed theory would have been more than 'shocking'. It would have been a scandal. So, instead, Venter and his colleagues went on to describe how they would develop new technologies that would enable researches to read the 'Book of Life' and thereby describe the most complex diseases and behaviours in terms of causal genes. In other words, the HGP leaders were saying that, in spite of the surprises, genetic explanations would be found as promised. (Strohman, 2002:1)

\section{Focus on Internal Rather than External Factors}

With the advent of genetic explanation of diseases, the external environment became progressively less important and the clinical focus shifted entirely to the internal environment. The primacy of the gene on the causation of disease has been a key element of the discourse surrounding the 'gene model'. This was even more restrictive in 'pre-genome' times because even the internal environment surrounding genes and their interaction with other genes was also largely absent from the discourse about their causal power. Even at the biological level, genetic elements are only one aspect of biological regulation. These interactions at the biological level were not present in most representations of the genetic basis of disease which concentrate on the causal power of genes ignoring their relationship with the rest of the organism. The human geneticists Scriver and Waters explain how this assumption ended up affecting their research. They 
expressed their expectations as follows:

(...)it was a hope that delineation of genotypes [as in the human genome project] with new methods for the detection of mutations would enable the prediction of variant phenotypes; in the case of human genetic disease, this would have added value to prognosis and treatment' (quoted in NeumannHeld, 2001: 10).

But this was not the case, as Scriver and Waters themselves admitted. Even for the case of classic monogenetic autosomal recessive diseases - such as the case of phenylketonuria (PKU) which they study - "expectations for a consistently close correlation between the mutant genotype and variant phenotype have been somehow disappointed" (Neumann-Held, 2001). This, they argue, was mainly due to the fact that the experiments were performed under highly controlled conditions but in reality "genomes function in vivo, where much more than the major gene is expressed and where the whole organismal phenotype is more than the sum of its parts..." (Neumann-Held, 2001)

The new science of genomics introduces a difference with respect to genetics in that it looks at the collection of all the genes in an individual and studies how those genes operate together. Genomics, however, stills focuses on internal factors only, if certainly less than genetics previously did, but still environmental and other factors extremely important in many of the diseases genomics is looking at, such as cancer or obesity, seem to lose their weight under the concept of genetic disease.

As Petersen (2002:25) highlights, it is acknowledged that much genetic variation may go unrecognized and remain relatively innocuous unless the individual is exposed to some critical environmental agent. This role of the environment, however, tends to be either put in second place, downplayed or completely ignored in the different variants of the gene model of disease. We have to acknowledge nonetheless, that recently there have been some attempts to soften this genetic determinism and more often allusions are made in both scientific and popular discourse to other factors (environment, diet, life-style, etc) influencing disease. However, these have been minor and not very successful in overriding the focus on genes as the locus of disease. In fact, some argue (as we will see below) that this phenomenon has been more of a narrative strategy to avoid the strong criticism of determinism than any real commitment to take these factors into consideration and therefore, does not actually challenge the model.

Adam Hedgecoe (2001) explores how scientists attempt to construct schizophrenia as a genetic disease using various discursive strategies. Hedgecoe argues that even on the new accounts where non-genetic factors are accepted, still the discourse about schizophrenia is constructed to prioritize genetic explanations, using a narrative he terms as 'enlightened geneticisation'. Geneticists use enlightened geneticisation to subtly privilege genetic explanations without succumbing to hard-line determinism. A central theme to the narrative, Hedgecoe stresses, is the presentation of current genetic thinking as reasonable, non-extremist, and accepting a role for non-genetic factors in schizophrenia causation. "Thus, although the narrative accepts some role for non-genetic causation, it 
is presented as a minor factor in comparison with the regular, specific nature of genetic causation" (Hedgecoe, 2001: 885).

In a different paper Hedgecoe (2003) looks at how the disease category of cystic fibrosis has been re-constructed along genetic lines to incorporate now neighbouring conditions that were previously considered separate. He argues that this redefinition conflicts with clinician's actual diagnostic practice and highlights the confusion and uncertainty that the introduction of genetic explanations can produce. (Hedgecoe, 2003:51). Sharer et al. (1998, quoted in Hedgecoe, 2003: 61) present the concerns of some in the cystic fibrosis medical community when they claim that far from improving diagnosis, the discovery of the CFTR-gene (i.e. the geneticization of CF) has meant that the definition of cystic fibrosis has become progressively hazier. There is a tension between the clinical context and the genetic reclassification. "Because the phenotypic spectrum that may now legitimately be called cystic fibrosis has become so large, it is unhelpful in the clinical context" (Sharer et al, 1999, quoted in Hedgecoe, 2003: 61). This highlights that not only every time more conditions are added to the genetic category, but also that those already considered genetic are continuously growing to the point of becoming ineffective. Accordingly, Colin et al. (1996) argue that there is a tension between clinicians, who doubt the pragmatic usefulness of the new geneticized classification system in CF, and researches that seem to be pushing it. Moreover, not only might clinical practice be affected by the geneticization of CF, but as Hedgecoe stresses, this also concerns concrete points of health policy.

We can perceive this 'enlightened geneticisation' taking place in a recent article appearing in Nature and written on behalf of the US National Human Genome Research Institute. The article was published on $24^{\text {th }}$ April 2003, just a few days after the publication of the completion of the HGP. Its title was "A Vision for the Future of Genomics Research" and within a 'genomics to biology; genomics to health and genomic to society' three-fold theme, it detailed a myriad of research opportunities for the new genome era. Here, the authors mention the importance of non-genetic factors in common diseases, only to emphasis the need for further genetic research.

Genetics seek to correlate variation in DNA sequence with phenotypic differences (traits). The greatest advances in human genetics have been made for traits associated with variation in a single gene. But most phenotypes, including common diseases and variable responses to pharmacological agents, have a more complex origin, involving the interplay between multiple genetic factors (gene and their products) and non-genetic factors (environmental influences). Unravelling such complexity will require both a complete description of the genetic variation in the human genome and the development of analytical tools for using that information to understand the genetic basis of disease. (Collins et al., 2003: 839).

Here, they explain how diseases are products of a multitude of factors; genes, their products and the environment. This seems to transcend genetic determinism since environment and other factors are included in the equation of disease causation. In the next sentence, 
however, when they go on to explain what is needed to understand diseases better, the role of the environment no longer enters the equation; the human genome and analytical tools to unravel the information coming from the same, are the only two factors mentioned as needed to unravel 'such complexity', the complexity itself being reduced to the genetic basis of disease. To understand the complexity causing disease they in fact ignore it by reducing it to genomics.

Further on they argue: "the genome is a dynamic structure, continually subjected to modification by the forces of evolution" (Collins et al., 2003: 839). This is a point certainly worth making since the popular image of the genome as a 'blueprint' seems to impose a static dimension which represents the genome as a stable entity. Accordingly, they argue that "mutation both drives longterm evolutionary change and it is the underlying cause of inherited disease' and that 'our understanding of DNA mutation and repair, including the important role of environmental factors, is limited" (Collins et al., 2003: 839). Here again they acknowledge, with even stronger claims, the vital role of the environment, at the same time acknowledging their limited knowledge about its input into disease. This acknowledgement, however, does not drive the authors into suggesting that because of its importance (and the recognised ignorance about it) the environment's role should be further studied. Instead, their next sentence argues: "genomics will provide the ability to substantively advance insights into evolutionary variation, which will, in turn, yield new insights into the dynamic nature of genomes in a broader evolutionary framework" (Collins et al., 2003: 839). How the study of genomics alone is going to throw light on the environmental part of disease causation and 'the important role of environmental factors on DNA mutation and repair' is never mentioned. We are left to believe that with further study of the genome, knowledge about other factors that are by their own admittance very important and so far limited will somehow simultaneously arise.

Thus, from popular representation to even some found on 'expert' journals such as Nature, the internal environment is clearly prioritised in explanations of disease causation. The role of the external environment seems more like a token that is introduced at some stage of the article but is almost never seriously analysed. Non-genetic factors are mentioned in passing while concentrating on the all-important role of the genes in causing a large number of diseases.

\section{Metaphor of the Body as Machine}

Through this metaphor, the body is conceived as made up of repairable and replaceable parts where problematic functioning can be identified and remedied by altering or replacing parts. Again, we can see this metaphor working in the 'gene model'. The discourse of genetic causation of disease is intimately linked with the idea of genetic therapy whereby genes are manipulated, repaired or deleted, to cure or prevent disease. This has not suffered any major changes due to the shift to genomics, but it will probably become even more prevalent if the concept of genetic disease with its assumptions of 'faulty genes' continue to prevail and new technologies are devel- 
oped to replace them. Martin (1999:517) argues that there has been "a shift in the field of gene therapy - from accounts based on classical genetics and the inheritance of deleterious genes, to one which explains many common acquired pathologies in terms of error in the way genes are regulated".

At the moment, however, genetic therapy does not seem to have any major relevance for clinical practice. The idea of genetic therapy formed part of the discourse creating the gene model from the beginning and in turn, the fact that we understand these diseases as genetic will make us look for genetic therapies to cure them. As Martin (1999) argues, the introduction of new technologies in genetics is also closely linked to the construction of new accounts about the origins of disease. Arguably, how we conceive disease will make us look in certain directions for their cure and prevention. If we follow the gene model, this will undoubtedly be the place we will be expecting help from and, in the process, if we are not careful, failing to see all those other places that hold key answers for most diseases.

In this respect, Baird (2002) argues that the theory that it will be possible to 'fix' disease by 'fixing' genes sounds plausible and it is easily understood by the public and the media. This she says, however, is an approach that has not been successful, not even for single disorders where the gene has been known and identified for many years (we do not have effective gene based therapy even for sickle-cell disease, CF or muscular dystrophy). If applied to common diseases (diabetes, heart disease, breast cancer, depression, etc) where multiple genes, as well as environment are inter- acting, this approach is even less likely to be effective. Furthermore, Baird (2002:526) stresses that, 'framing ill health as genetic and promoting individual genetic or pharmacological solutions pushes the problem back to the individual, so genetic explanations are attractive to those who do not want to deal with complex social and economic determinants of health'.

In the same article in The Guardian that I referred to earlier, Sir John Sulton, moves from challenging determinism to explaining how the small number of genes confirmed Darwin's theory of evolution.

Is the unity of life, of nature being conservative, or the idea of the Blind Watchmaker-the notion of evolution as a constant reworking or random recombining of parts. You convert your Austin 7 into a Mercedes, but basically it is the same underneath.

This metaphor of the body-as-machine grows from the hierarchic component of reductionism that sees the body as earthy, comparatively simple and predictable, manipulable, and controllable (Gordon, 1988; Kirmayer 1988). It objectifies the body and supports the notion that being made up of parts that can be "fixed" or "replaced," like a car's. As we mentioned earlier this is a very powerful assumption underlying the concepts of genetic disease and genetic therapy.

\section{Discussion}

The publication of the HGP and the move to more complex genomics has not eliminated the previous determinism that genetics represented. Even if scientists are in their actual work looking at a broader range of factors and taking 
now into account more the interactions within the genome and the organism as a whole, the social representation of the relationship between genes and disease does not seem to have changed as much as one would expect, and published reports still contribute to strengthen the belief in the all-encompassing explanatory power of genes. The knowledge of the genes and the genomes is still presented as the most important source for understanding diseases in both the public and the scientific discourse. And these discourses, as Hubbard and Wald (1999) argue, fuel the widely held perception that our health problems originate inside us and draw attention away from outside factors that need to be addressed.

In spite of empirical evidence to the contrary more causal power is still attributed to the genes in the development of a disease. Therefore, the research on genes is still given higher priority than that on other mechanisms which might participate in the causation of disease.

To conclude, I would like to stress that this criticism of the geneticisation of disease and the expansion of the concept of genetic disease is not just a matter of semantics, but it has real consequences for real people. Both at societal and at individual levels the definition of what constitutes an illness and which type of illness it is, affects much more than just language. The emphasis upon disease as result of something we have inside (genes) that occurs within an individual also produces a tendency to locate responsibility for illness in the individual. It is thus one form of blaming the victim; a type of legitimisation that argues that the victim rather than the agent of misfortune was actually responsible for the occurrence. This approach to dis- ease, as a condition of the sick individual thus depoliticises illness causation (McKinlay, 1994). By contrast, adequate social policies for prevention require an awareness of the social and environmental causes of illness and the social context of the sick person.

It is also argued that the increasing use of genetic information is driving health-care attention toward genes and away from other issues (both social and environmental as well as other medical perspectives) and contributing to a medicalisation that is making increasing numbers of people "patients" of future diseases that may, or may not, befall upon them (Melzer and Zimmern, 2002).

The goal of avoiding the onset of common multifactorial disorders is obviously a worthy one. However, a genetic approach to the common diseases that makes up most of the population disease burden is inherently limited in what it can achieve (Baird, 2001; Beaglehole, 2001). In the last several decades, mortality from cardiovascular disease has fallen by more than 40 percent-not as a result of identifying individuals who are at genetic risk, but largely because of social changes such as increased physical activity, better dietary and smoking habits, and socioeconomic changes. It would be more effective to devote resources to changing and improving the environment for everyone, than to invest societal resources in susceptibility testing. (Baird, 2002:526). This needed transition from a gene model with its imbedded genetic determinism and reductionism to a new approach where diseases are looked at in their whole complexity and dynamics has not yet occurred and it is indeed much needed. 


\section{Acknowledgement}

I would like to thank the European Union for granting me a Marie Curie Fellowship that funded my research stay in Helsinki.

\section{References}

Baird, P.A.

2002 "Identification of Genetic Susceptibility to Common Diseases: the case for regulation." Perspectives in Biology and Medicine 45,4: 516-528.

Beaglehole, R.

2001 "Global Cardiovascular Disease Prevention: Time to Get Serious". The Lancet, 358: 661-663.

Colin, A., Sawyer S., Mickle J., Oates, R., Milunsky A. \& Amos, J.

1996 "Pulmonary function and clinical observations in men with congenital bilateral absence of the vas deference." Chest 110,2:440-5.

Collins, F.

1999 Shattuck Lecture-Medical and societal consequences of the Human Genome Project. New England Journal of Medicine 341:28-37.

Collins et al.

2003 "A vision for the future of genomic research." Nature 422: 835-847.

Conrad, P.

1999 "A mirage of genes." Sociology of Health \& Illness. 21,2: 228-241.

Dubos, R.

1959 Mirage of Health. NewYork: Harper and Row.

Freund, P. \& McGuire, M.

1995 Health, Illness, and the Social Body. New Jersey: Prentice Hall.

Gordon D.

1988 "Tenacious Assumptions in Western Medicine." in Lock and Gordon (eds.) Biomedicine Examined. Dordrecht: Kluwer Academic Publishers.

Guttmacher, Alan E. \& Collins, F.

2002 Genomic Medicine - A Primer. 347: 1512-1520.
Hedgecoe, A.

2003 "Expansion and uncertainty: cystic fibrosis, classification and genetics" Sociology of Health and Illness. 25,1: 5070.

2001 "Schizophrenia and the Narrative of Enlightened Geneticization" Social Studies of Science. 31,6: 875-911.

Hieter, P. \& Boguski, M.

1997 Functional Genomics: It's all how you read it. Science 278:601-602.

Hubbard, R. \& Wald, E.

1999 Exploding the Gene Myth. Boston: Beacon Press.

Keating, P. \& Cambrosio, A.

2001 "The New Genetics and Cancer: The Contributions of Clinical Medicine in the Era of Biomedicine." Journal of the History of Medicine and Allied Sciences. 56,4: 321-352

Keller, E.F.

1992 "Nature, Nurture and the Human Genome Project." in D. J. Kevles and L. Hood. The Code of Codes. London: Harvard University Press.

Kirmayer L.

1988 "Mind and Body as Metaphors: Hidden Values in Biomedicine." in M. Lock and D. Gordon (eds.). Biomedicine Examined, Boston: Kluwer Academic Publishers.

Lewis, R.

2000 "Keeping Up: Genetics to Genomics in Four Editions" The Scientist 14, 15:46.

Lippman, A.

1991 "Prenatal Genetic Testing and Screening: Constructing Needs and Reinforcing Inequalities" American Journal of Law and Medicine. 17, 1\&2:15-50.

Magnus, D.

(n/d) "Gene Therapy and the Concept of Genetic Disease." Genetics and Ethics: a Global Conversation. On-line publication by University of Pennsylvania, http://www.med.upenn.edu/bioethic/ genetics/articles12.gen.disease.html.

Martin, E.

1991 "The egg and the sperm: how science has constructed a romance based on stereotypical male-female roles." Signs. 16,31:485-501. 
Martin, P.

1999 "Genes as drugs: the social shaping of gene therapy and the reconstruction of genetic disease." Sociology of Health \& Illness. 21, 5: 517-538.

Melzer, D. \& Zimmern, R.

2002 "Genetics and medicalisation" British Medical Journal. 324:863-864.

Nelkin, D.

1987 Selling science: How the press covers science and technology. New York: W. H. Freeman\& Co.

Neumann-Held, E.

2001 "Can it be a 'sin' to understand disease? On 'genes' and 'eugenics' and an 'unconnected connection". Medicine,

Petersen, A. Health Care and Philosophy. 4: 5-17.

2002 "The new genetics and the media" in A. Petersen and R. Bunton. The new genetics and the public's health. London and New York: Routledge.

2001 "Biofantasies: genetics and medicine in the print news media". Social Science and Medicine, 52: 1255-1268.

Scriver, C.

2001 Genetic Disease: An Orphan in Canadian Healthcare. Canadian Journal of Policy Research 2:113-8.

Strohman, R.

2000 "Genetic determinism as a failing paradigm in biology and medicine" in M. J. Schneider and D. Stokols Promoting Human Wellness. London: University of California Press.

2002 "Beyond Genetic Determinism" Pressing Times, Spring. http://www. mindfully.org/GE/GE4/Beyond-Genetic-Determinism-Apr02.htm

Thieffry, D. \& Sarkar, S.

1999 "Postgenomics? A conference at the Max Planck Institute for the History of Science in Berlin" BioScience 49,3.

Van Dijk, T.

1998 Imagenation: Popular images of genetics. New York: New York University Press.

Yoxen, E.

1984 "Constructing Genetic Disease" in T. Duster and K. Garett (eds) Cultural Perspectives on Biological Knowledge. Norwood, NJ.: Ablex.
Sara Melendro-Oliver

Egenis

ESRC Centre for Genomics in Society

University of Exeter, UK

S.Melendro-Oliver@exeter.ac.uk 